\title{
New Ultrasound System for Bone Assessment
}

\author{
Jonathan J. Kaufman ${ }^{* a, b}$, Gangming Luo ${ }^{c}$, David Conroy ${ }^{\mathrm{d}}$, William A. Johnson ${ }^{\mathrm{e}}$, Ronald L. Altman ${ }^{\mathrm{e}}$, \\ Robert S. Siffert ${ }^{\mathrm{b}}$ \\ ${ }^{a}$ CyberLogic, Inc., 611 Broadway, Suite 707, New York, NY 10012 \\ ${ }^{b}$ Dept. of Orthopaedics, The Mount Sinai School of Med., One Gustave L. Levy Place, NY, NY \\ ${ }^{c}$ New York Department of Veterans Affairs Medical Center \& Department of Rehabilitation \\ Medicine, The New York University Medical Center, New York, NY \\ ${ }^{\mathrm{d}}$ Design at Work, 361 Parkside Avenue, Brooklyn, NY 11226 \\ ${ }^{\mathrm{e}}$ Techen, Inc., 115 Cedar Street, Milford, MA 01757
}

\begin{abstract}
This paper reports on a new ultrasound device for noninvasive assessment of bone. The device, known as the QRT 2000 - for Quantitative Real-Time -- is entirely self-contained, portable, and handheld. The $Q R T 2000$ is powered by 4 "AA" rechargeable batteries and permits near real-time evaluation of a novel set of ultrasound parameters and their on-line display to the user. The parameters have been studied both in vitro and clinically with a laboratory unit that measured the calcaneus in through transmission and computed the ultrasound features off-line. The data related the ultrasound parameters to the bone mineral density (BMD) of the calcaneus, spine and hip, as determined by x-ray absorptiometry, and demonstrated that the parameters were superior to the standard ones known as BUA and SOS (broadband ultrasound attenuation and speed-of-sound, respectively). The QRT 2000 was then constructed to compute the same parameters; however as noted above it does this in near real-time and provides visual feedback to the user while the measurements are being made. The compactness and portability of the unit make it also ideal for spaceflight applications. Finally, the QRT 2000 was designed to be manufactured at relatively low cost, and therefore should enable the significant expansion of quantitative ultrasound measurements to, for example, primary care physicians in this country and abroad, and including for use in the developing world.
\end{abstract}

Keywords: Ultrasound, bone, BMD, calcaneus, heel, osteoporosis, mean frequency, real-time, portable, hand-held

\section{INTRODUCTION}

This study has as its long term objective establishing ultrasound as a safe, effective, and non-invasive method for accurately and precisely assessing bone strength and fracture risk, important components in the clinical management of metabolic bone diseases, especially osteoporosis. Currently, bone is mainly assessed using one of the x-ray densitometric methods, for example with dual energy x-ray absorptiometry ${ }^{1}$. While providing reasonable indications of bone integrity, these $\mathrm{x}$-ray densitometric techniques are relatively expensive, expose the patient to ionizing radiation, and are not able to characterize all of the variability observed in bone strength or accurately predict the occurrence of fractures in individuals. As is well known, mass based methods do not provide any information on other factors known to be associated with bone strength, such as bone architecture and tissue quality $y^{2-3}$. These various shortcomings of current densitometric techniques have led to the search for more effective bone assessment methods. One such technique is based on ultrasound, which offers several potential advantages for assessing bone strength and fracture risk ${ }^{4}$. First, ultrasound is non-ionizing and relatively easy to generate and detect; thus, it would be an attractive technique in terms of its cost and safety for assessing bone integrity. The lower cost of an ultrasonic assessment system is especially important in view of the need to contain ever-increasing health care costs. Second, ultrasound may be able to estimate bone mass and thus provide equivalent information to current densitometric bone scanners. Finally, since ultrasound is a mechanical wave and interacts with bone in a fundamentally different manner than the electromagnetic radiation of densitometric scanners, it may be able to provide more accurate estimates of bone strength compared with mass based predictions ${ }^{5}$. The availability of such a technique would have wide use in clinical management of metabolic bone diseases, and in addition could help foster the development of large scale screening programs.

\footnotetext{
*E-mail: jjkaufman@ @yberlogic.org; Phone: 212-260-1351; Fax: 212-260-1353; Web: http://www.cyberlogic.org
} 
Notwithstanding the above, ultrasound has not yet fulfilled its promise in noninvasive bone assessment. Current devices suffer from several shortcomings. The first shortcoming is the relatively complex implementations associated with current ultrasonic techniques typically are based on analog-to-digital conversion and storage of the ultrasonic signals, off-line processing and incorporation of a computer as an integral part of the overall device. Besides increasing the costs of the ultrasound device (and test), the associated complexity also reduces device portability and ease of use. Another shortcoming is the presence of numerous experimental artifacts associated with the signal processing used in present ultrasound assessment devices, which can lead to significant reductions in diagnostic accuracy and precision. To address these shortcomings, we have developed an ultrasonic system which is based on a new set of ultrasound parameters that are more resistant to various experimental artifacts and which appear to provide enhanced accuracy in assessing bone. The device, known as the QRT 2000, is entirely handheld and portable and uses 4 "AA" rechargeable batteries. It provides near real-time feedback to the user -- which can be useful in repositioning the transducers - through a graphical display showing the parameters as they care computed on-line. The $Q R T 2000$ is also expected to have a significant advantage over other devices because of its very competitive pricing (\$5000), about half the cost of present ultrasound devices and about 5-20 times less than x-ray bone densitometers.

\section{SIGNAL PROCESSING AND ULTRASOUND PARAMETERS}

The use of ultrasound to quantitatively assess bone is based on a straightforward principle (hypothesis), namely that different bones (with distinct bone densities and strengths will affect a propagating ultrasound wave in different ways. Therefore, by measuring these changes, that is, by assessing the changes induced in the ultrasound waveform after it has propagated through a given bone, one may try to ascertain its properties. If $\mathrm{V}_{\mathrm{i}}(\mathrm{f})$ is the Fourier transform of the signal which is input to the bone being interrogated, and $\mathrm{V}_{\mathrm{o}}(\mathrm{f})$ is the Fourier transform of the signal after it has propagated through the bone, then the acoustic transfer function (of a bone, for example) can be expressed as

$\mathrm{H}(\mathrm{f}) \quad=\mathrm{V}_{0}(\mathrm{f}) / \mathrm{V}_{\mathrm{i}}(\mathrm{f})=\mathrm{e}^{-\alpha(\mathrm{f})-\mathrm{j} \varphi(\mathrm{f})}$

In the above equation, $\alpha(\mathrm{f})$ and $\varphi(f)$ are the attenuation and phase functions, respectively, associated with the bone $\mathrm{e}^{6}$ Generally, the attenuation is modeled as an affine function and the slope of this function is referred to as BUA, which is used to characterize the bone. The phase function denoted by $\varphi(f)$ determines the velocity at which the ultrasound wave propagates through the bone ${ }^{7}$. The phase function allows the computation of the both the phase and group velocity, although most present devices use a time of flight measurement (e.g., SOS) which does not necessarily correlate well with either the phase or group velocity. It should also be noted that the above characterization does not take into account the presence of multiple modes of propagation, including slow and fast Biot modes or multi-path signals due for example to reflections in the soft tissue layers overlying the bone. The algorithms used in this study incorporate a more general attenuation and phase function; specifically the attenuation and phase are modeled by:

$\alpha(\mathrm{f})=\mathrm{A}_{0}+\mathrm{A}_{1} \mathrm{f}+\mathrm{A}_{2} \mathrm{f}^{2}$

$\varphi(f)=\varphi_{0}+\varphi_{1} \omega$

Now, assume that the source pulse can be modeled as a sinusoid with a Gaussian envelope:

$\mathrm{v}_{\mathrm{i}}(\mathrm{t})=\exp \left[-\sigma \mathrm{r}^{2} \mathrm{t}^{2} / 2\right] \cos \left(\omega_{\mathrm{r}} \mathrm{t}+\varphi_{\mathrm{r}}\right)$

Then it can be shown that the received waveform, vo(t), after propagating through a medium having the attenuation and phase as given in the above two equations is given by

$\operatorname{vo}(\mathrm{t})=\mathrm{k} \exp [-(\mathrm{t}-\tau) 2 / \Delta 2 \cos (\omega \mathrm{t}+\varphi \mathrm{p})$

(that is, another sinusoid with a Gaussian envelope) where 


$$
\begin{aligned}
& \mathrm{A}_{2}=\Delta^{2} / 4-1 /\left(4 \sigma \mathrm{r}^{2}\right) \\
& \mathrm{A}_{1}=(\omega \mathrm{r}-\omega) /\left(2 \sigma \mathrm{r}^{2}\right)-2 \omega \mathrm{A}_{2} \\
& \left.\mathrm{~A} 0=-\ln (\mathrm{k})-(\omega \mathrm{r}-\omega)^{2} /\left(4 \sigma \mathrm{r}^{2}\right)-\ln \sigma \mathrm{r}^{4} \Delta^{4}-\mathrm{A} 1 \omega \mathrm{r}-\mathrm{A} 2 \omega^{2}\right) \\
& \varphi 1=\tau
\end{aligned}
$$

The received ultrasound signal is processed to obtain the relative mean frequency (RMF), the relative pulse width (RPW) and envelope velocity (EV), which are given (approximately) by:

$$
\begin{aligned}
& \mathrm{RMF} \approx \omega / \omega \mathrm{r} \\
& \mathrm{RPW} \approx \Delta \mathrm{r} / \Delta
\end{aligned}
$$

and

$$
\mathrm{EV} \approx 1 / \tau
$$

These parameters may be estimated using the analytic signal obtained via the Hilbert transform, or more simply through rectification and low pass filtering and zero crossing analysis ${ }^{8-10}$. It should be noted that the above parameters contain an equivalent set of information as the parameters of the transfer function itself, and that they potentially contain more information than BUA and SOS. It should also be pointed out that although the analysis was based on a Gaussian source wavelet, the parametric approach can still be used in cases where the Gaussian assumption is not strictly true. In such cases, it is assumed that the parameters nevertheless capture the essential features of the transfer function, as long as the source pulse is not "too far" from Gaussian.

In addition to the above 3 parameters, another parameter was defined: the relative energy (RE), which measures the total amount of energy transmitted through the heel, relative to the known reference or input signal. Thus the three parameters, namely RMF, RPW, and RE characterize the average frequency content in the ultrasonic signal, the inverse bandwidth of the signal, and the total energy that is received by the receiver, all relative to the source waveform, respectively. The EV is the speed with which the signal energy is propagated through the heel. Please note that the RMF is expressed in the following as mean instantaneous frequency (MIF), but is equivalent to RMF since the same source waveform was used in all cases.

\section{EXPERIMENTAL STUDIES}

The two studies had as their objective to examine the potential usefulness of the above ultrasonic processing method. The technique was evaluated using both an in vitro human bone study and a clinical study. The in vitro study was designed to develop a better understanding of the ultrasonic measurement techniques under well controlled conditions. The clinical study was carried out to determine how the ultrasound techniques performed under "real-world" conditions.

\subsection{In Vitro Study}

The experiment consisted of acquiring 99 human calcanei which had been previously cleaned of all soft tissue and for which no identifying information was available. A $1.5 \mathrm{~cm}$ cylindrical core was extracted from the posterior portion of each calcaneus in the medial lateral direction. The cores, with their cortical shells intact, were then saturated with a buffered solution and ultrasonically measured using a $1 / 2$ " diameter $1 \mathrm{MHz}$ broadband transducer pair (Model V303, GE Panametrics, Waltham, MA). Three separate acquisitions were obtained and the results of each averaged together in order to evaluate the mean ultrasonic feature for each sample. The relative precision of each ultrasonic feature was also evaluated. Their bone mineral densities in grams per square centimeter $[\mathrm{g} / \mathrm{cm} 2]$ were determined by single photon absorptiometry (SPA). The ultrasonic measurements were compared to the densities, using univariate and multivariate linear regressions. In addition, a comparison was made between the "standard" ultrasound parameters, broadband 
ultrasound attenuation (BUA) and ultrasound velocity (SOS), and the new ultrasound feature set, in terms of their respective correlations with BMD.

Table I displays a statistical summary of all the data obtained in this in vitro study. As may be seen the BMD varied between a minimum value of $0.158 \mathrm{~g} / \mathrm{cm} 2$ and a maximum value of $0.635 \mathrm{~g} / \mathrm{cm} 2$, with a mean value of $0.3618 \mathrm{~g} / \mathrm{cm} 2$. Table II displays the linear correlation coefficients for all the ultrasound parameters and bone sample length with respect to BMD. The single best predictor of calcaneal bone mineral density was the mean instantaneous frequency (MIF), providing a correlation coefficient of $\mathrm{R}=0.8 \quad(\mathrm{P}<0.0001, \mathrm{~N}=99)$, with a standard error of the estimate or rootmean-square (RMS ) error of $0.06 \mathrm{~g} / \mathrm{cm} 2$. Fig. 1 shows the MIF feature (i.e., 1$\mathrm{MIF} / \mathrm{MIF}_{\mathrm{R}}$, where $\mathrm{MIF}_{\mathrm{R}}$ is the MIF associated with a reference ultrasound signal) as a function of BMD. The MIF was

\begin{tabular}{|l|l|l|l|l|}
\hline $\begin{array}{l}\text { TABLE I: Statistical } \\
\text { Summary: In Vitro } \\
\begin{array}{l}\text { Human Calcaneal } \\
\text { Bone Samples (N=99) }\end{array}\end{array}$ & Mean & Std. Dev. & Max & Min \\
\hline BMD [g/cm $\left.{ }^{2}\right]$ & 0.362 & 0.0875 & 0.635 & 0.158 \\
\hline MIF & 0.372 & 0.0846 & 0.545 & 0.143 \\
\hline RE & 0.0233 & 0.0315 & 0.228 & 0.00147 \\
\hline RPW & 1.297 & 0.136 & 1.764 & 1.095 \\
\hline EV [m/s] & 1528 & 21.7 & 1607 & 1488 \\
\hline BUA [dB/MHZ] & 43.2 & 13.0 & 80.2 & 13.2 \\
\hline SOS [m/s] & 1702 & 43.6 & 1847 & 1601 \\
\hline
\end{tabular}
then combined with three other ultrasound features and with the bone sample length, ds, in a multivariate linear regression. The four ultrasound features, i.e., MIF, RPW, RE and EV are the new set of ultrasound parameters which were evaluated to see if they are an effective means for assessing bone. The combination of these four features with ds, produced a correlation coefficient of $\mathrm{R}=0.85$, and an RMS error of only $0.045 \mathrm{~g} / \mathrm{cm} 2$, representing about a 25 percent improvement in the quality of the BMD estimate over that obtained with MIF alone (Fig. 2). This error of $0.045 \mathrm{~g} / \mathrm{cm} 2 \mathrm{is}$ a relatively small error in terms of screening for low bone mass, being only about 12 percent of the mean bone mass itself. A multivariate linear regression with BUA, SOS and $d_{s}$ produced an RMS error of $0.056 \mathrm{~g} / \mathrm{cm} 2$, more than a 24 percent deterioration in the quality of the estimate.

TABLE II

Linear Correlation Coefficients: In Vitro Human Calcaneal Bone Samples (N=99) Correlation with BMD

\begin{tabular}{|l|l|l|l|l|l|l|}
\hline$M I F$ & $R E$ & $E V$ & $R P W$ & $d_{s}$ & BUA & SOS \\
\hline 0.80 & 0.76 & 0.60 & 0.71 & 0.30 & 0.76 & 0.58 \\
\hline
\end{tabular}

TABLE III

RMS Errors: In Vitro Human Calcaneal Bone Samples ( $N=99)$

\begin{tabular}{|l|l|l|l|}
\hline & $\begin{array}{l}\text { Univariate Linear } \\
\text { Regression }\end{array}$ & $\begin{array}{l}\text { Multivariate Linear } \\
\text { Regression }\end{array}$ & $\begin{array}{l}\text { Multivariate } \\
\text { Linear } \\
\text { Regression }\end{array}$ \\
\hline Inputs & $M I F$ & $M I F, R P W, R E, E V, d_{s}$ & BUA, SOS, $d_{s}$ \\
\hline$R M S$ Error $\left[\mathrm{g} / \mathrm{cm}^{2}\right]$ & 0.06 & 0.045 & 0.056 \\
\hline
\end{tabular}




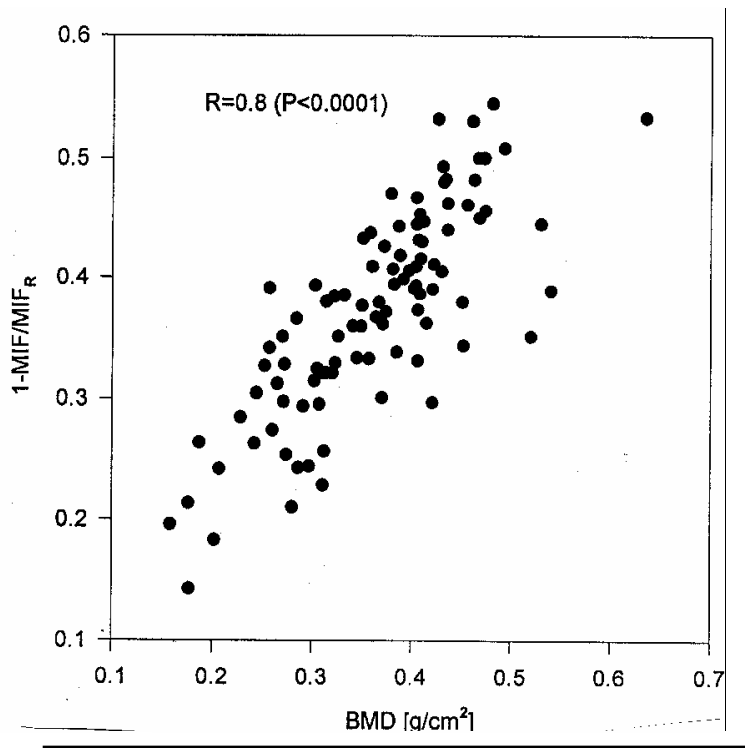

Figure 1. Plot of the mean instantaneous frequency parameter $1-\mathrm{MIF} / \mathrm{MIF}_{\mathrm{R}}$ as a function of bone mineral density (BMD), for the 99 calcaneal cylindrical bone samples. $\mathrm{MIF}_{\mathrm{R}}$ is the mean instantaneous frequency associated with the ultrasound reference signal.

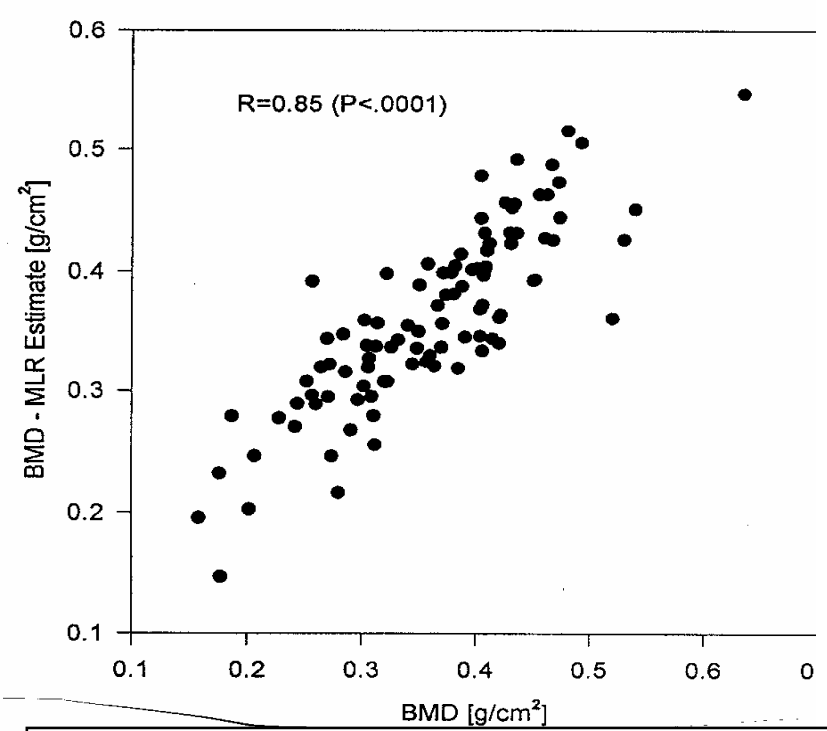

Figure 2. Plot of the multivariate linear regression estimate of bone mineral density vs. bone mineral density for the 99 calcaneal cylindrical bone samples. The linear multivariate regression estimate uses MIF, RPW, EV, RE and sample length, $\mathrm{d}_{\mathrm{s}}$, as independent variables.

Table III summarizes these results for the three BMD estimation methods. An estimate of the average precision of the ultrasonic features was also determined. We found precisions of $0.8 \%, 1.1 \%, 2.1 \%, 5.4 \%, 3.2 \%$ and $1.1 \%$ for the EV, RPW, MIF, RE, BUA and SOS ultrasound parameters, respectively.

TABLE III

RMS Errors: In Vitro Human Calcaneal Bone Samples ( $N=99)$

\begin{tabular}{|l|l|l|l|}
\hline & $\begin{array}{l}\text { Univariate Linear } \\
\text { Regression }\end{array}$ & $\begin{array}{l}\text { Multivariate Linear } \\
\text { Regression }\end{array}$ & $\begin{array}{l}\text { Multivariate } \\
\text { Linear } \\
\text { Regression }\end{array}$ \\
\hline Inputs & $M I F$ & $M I F, R P W, R E, E V, d_{s}$ & BUA, SOS, $d_{s}$ \\
\hline$R M S$ Error $\left[\mathrm{g} / \mathrm{cm}^{2}\right]$ & 0.06 & 0.045 & 0.056 \\
\hline
\end{tabular}




\subsection{Clinical Study}

A total of 165 female subjects, the majority of whom were referred by their physicians for bone densitometry to evaluate their fracture risk, served as the basis for our study. They ranged in age from 24 to 94 years with a mean age of 57.5. Of these 165 patients, 153 had spine density measurements, 154 had hip density measurements, and 146 had both spine and hip density measurements. The spine and hip bone mineral densities were measured using DXA with a Hologic QDR-2000 bone densitometer. Ultrasound measurements were obtained as follows. Two 0.5 " diameter 1 $\mathrm{MHz}$ coaxially aligned transducers (Panametrics V303) were placed in direct contact with the skin overlying the medial and lateral sides of the subjects' heels, with ample coupling gel to ensure

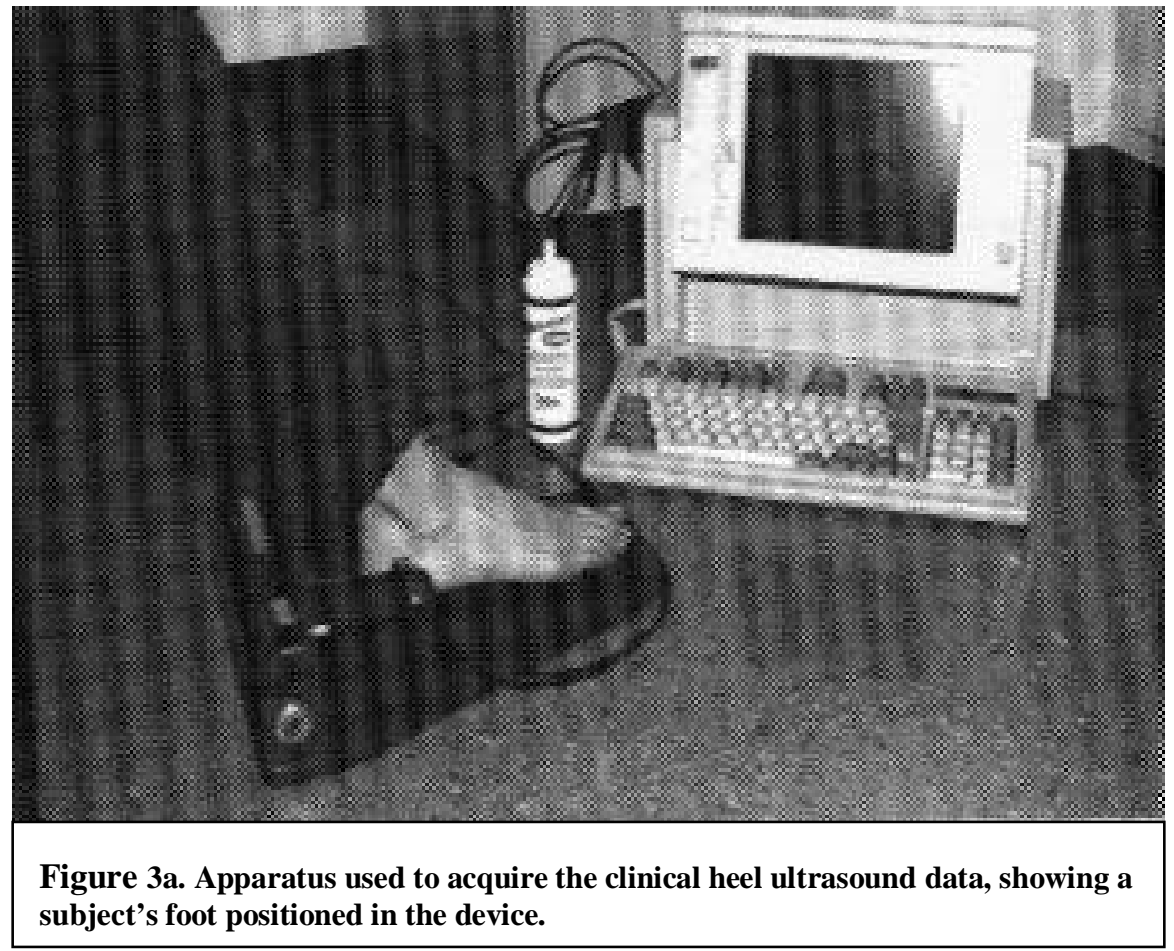
good conduction (Fig. 3a). One transducer served as a transmitter and the other as a receiver of the acoustic wave after it propagated through the posterior portion of the calcaneus (Fig. 3b) and overlying soft tissue.

Analogously with the in vitro study, the set of new ultrasonic parameters was evaluated, namely the mean instantaneous frequency (MIF), relative pulse width (RPW), envelope velocity (EV) and relative energy (RE). The standard ultrasonic features were also evaluated, i.e., BUA and SOS. As pointed out earlier, although SOS is commonly measured and reported, it is generally neither a phase nor group velocity. On the other hand, EV is the velocity associated with the rate at which information is propagated through the bone tissue. Three independent realizations of all the ultrasound parameters, obtaining by removing the heel completely and repositioning it for another ultrasound measurement, were averaged to obtain the mean values for each subject. An ultrasound signal which propagated through water only was also collected

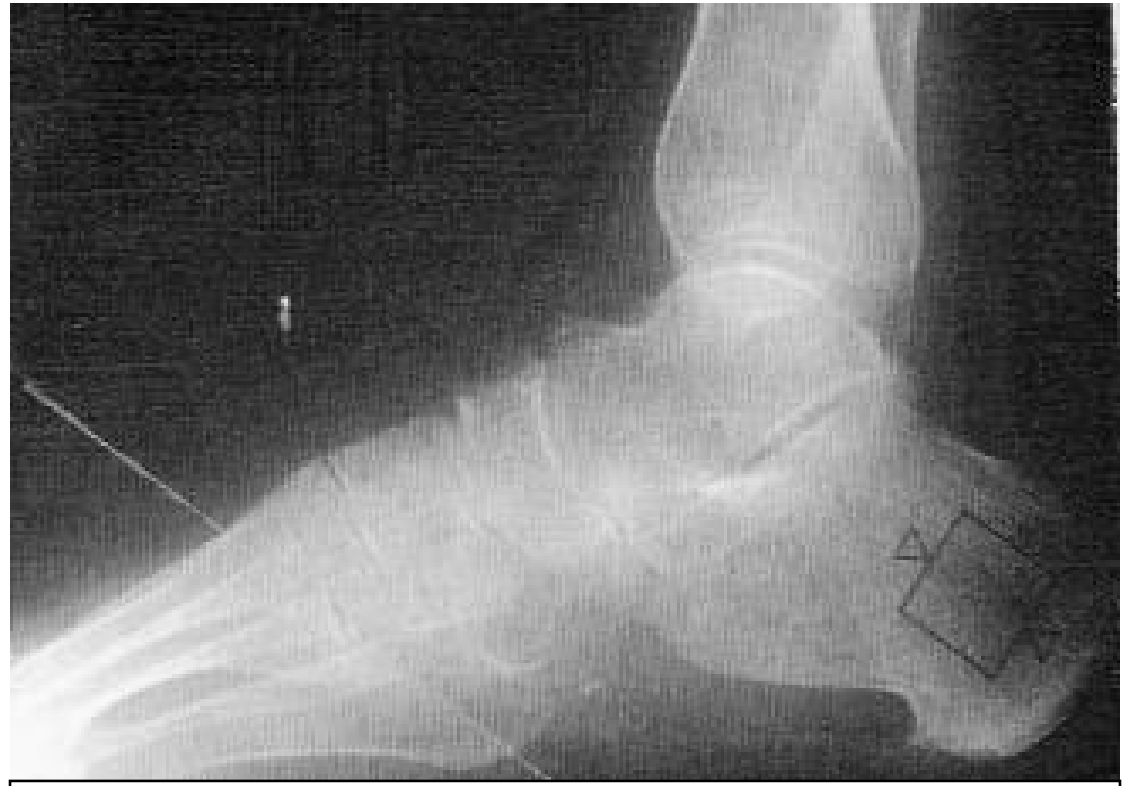

Figure 3b. Radiograph of the heel showing approximate location of the ultrasound measurements. [Figure courtesy of Dr. Mary Bouxsein.1

and served as a reference in the analysis. Table $\mathrm{V}$ summarizes the complete set of statistics for the clinical data. In this clinical data, for example, the spine BMD displays a little more than a three-fold variation. This is somewhat less than 
the four-fold variation expressed by the in vitro calcaneal samples. Table VI presents the linear correlation coefficients for the ultrasound parameters and age and weight with respect to BMD's of the lumbar spine, hip-neck and hiptrochanteric regions, respectively. As may be seen, the two best ultrasound features in terms of linear correlations are the relative pulse width (RPW) and mean instantaneous frequency (MIF), providing average correlations of 0.60 and 0.57 , respectively.

A multivariate linear regression was carried out for both the lumbar spine BMD and hip-neck BMD. Results for the hiptrochanteric BMD were almost identical to the hip-neck $\mathrm{BMD}$ and are not shown in the subsequent data. The independent variables were the four ultrasound features, namely, MIF, RPW, RE, and EV, together with each subject's age and weight. Correlation coefficients of $\mathrm{R}=$ 0.75 and $R=0.80$ were obtained, for the spine and hip-neck BMD's respectively. Fig. 4 shows the plot of the multivariate linear regression BMD estimate for the spine BMD, and Fig. 5 shows the plot of the multivariate linear regression BMD estimate for the hip-neck BMD. We also evaluated the correlation coefficients between estimates of spine and hip-neck BMD's based on BUA, SOS, age and weight; the correlation coefficients were 0.70 and 0.74 , respectively, for the spine and hip-neck BMD's, respectively. This represents a 12 percent and 16 percent deterioration in the standard error of the lumbar spine and hip-neck BMD estimates, respectively, when using the typical BUA and SOS ultrasonic parameters.

The excellent performance of these new ultrasonic parameters, particularly $M I F$ and $R P W$, is believed to be related to two main factors. The first deals with the information content in the parameters themselves. While standard ultrasonic techniques rely primarily on $B U A$, the use of both MIF and $R P W$ provides additional information related to the attenuation and phase response of the interrogated bone. No assumptions of linearity, are made, for example, with respect to the attenuation function. The second and perhaps equally important aspect of the MIF and other proprietary ultrasonic features is that they are nonlinearly related to the ultrasonic waveforms and less sensitive to various experimental artifacts, such as multiple reflections and interfering modes of propagation. A final advantage of the new parameters is their capacity to be implemented in real time and with relatively simple electronics. Besides allowing more reliable, easy to use, and more cost efficient devices, this capability also allows for the practical (but future) implementation of array based methods, which can lead to even more precise and accurate bone assessment

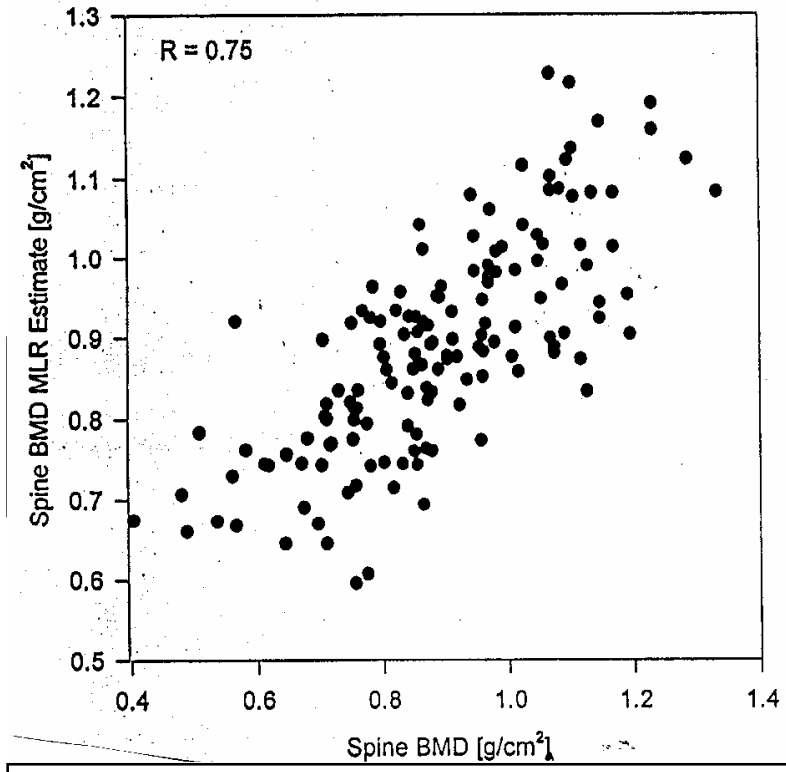

Figure 4. Multivariate linear regression estimate of the spine BMD based on ultrasonic measurements and subject age and weight vs. spine BMD determined by DXA.

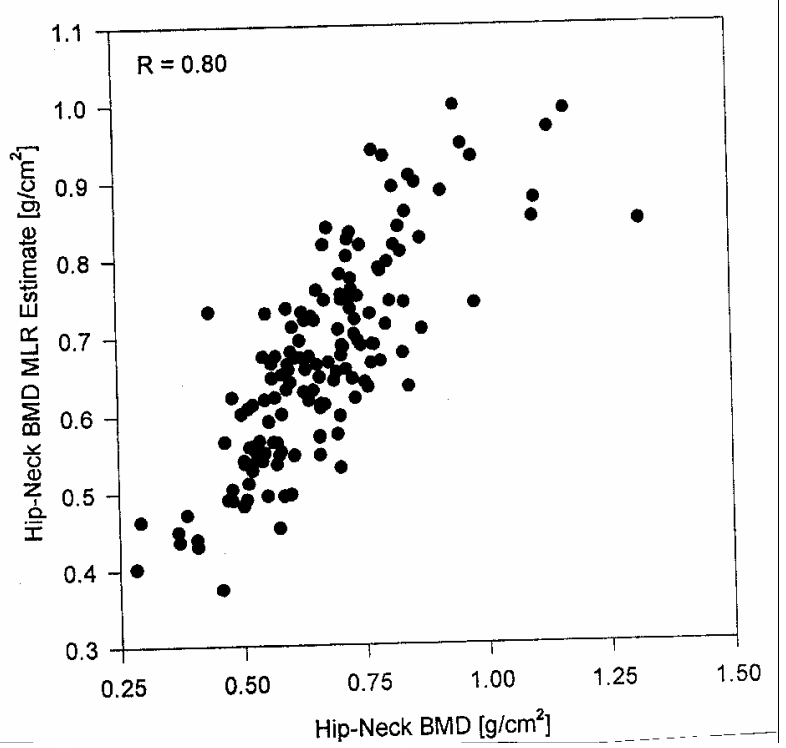

Figure 5. Multivariate linear regression estimate of the hip-neck BMD based on ultrasonic measurements and subject age and weight vs. hip-neck BMD determined by DXA. 
TABLE V

Statistical Summary: Clinical Heel Ultrasound Study

\begin{tabular}{|l|l|l|l|l|}
\hline & Mean & Std. Dev. & Max & Min \\
\hline $\begin{array}{l}\text { Lumbar Spine BMD } \\
{\left[\mathrm{g} / \mathrm{cm}^{2}\right]}\end{array}$ & & & & \\
\hline Hip-Neck BMD [g/cm $\left.{ }^{2}\right]$ & 0.665 & 0.162 & 1.32 & 0.404 \\
\hline Hip-Troch BMD [g/cm $\left.{ }^{2}\right]$ & 0.577 & 0.131 & 1.09 & 0.383 \\
\hline MIF & 0.572 & 0.0846 & 0.732 & 0.053 \\
\hline ER & $3.3 \times 10^{-3}$ & $4.0 \times 10^{-3}$ & $22 \times 10^{-3}$ & 0.407 \\
\hline RPW & 1.91 & 0.430 & 3.41 & $0.07 \times 10^{-3}$ \\
\hline EV [m/s] & 1505 & 25.3 & 1620 & 1.18 \\
\hline BUA [dB/MHZ] & 85.6 & 19.2 & 131 & 1460 \\
\hline SOS [m/s] & 1679 & 63.3 & 1902 & 45.6 \\
\hline
\end{tabular}

TABLE VI

Linear Correlation Coefficients: Clinical Heel Ultrasound Study -- Correlations with BMD

\begin{tabular}{|l|l|l|l|l|l|l|l|l|l|}
\hline & $M I F$ & $R E$ & $E V$ & $R P W$ & $d_{h}$ & Age & Weight & BUA & SOS \\
\hline Spine BMD & 0.56 & 0.42 & 0.54 & 0.60 & 0.003 & 0.55 & 0.37 & 0.54 & 0.49 \\
\hline Hip-Neck BMD & 0.59 & 0.45 & 0.52 & 0.63 & 0.06 & 0.63 & 0.43 & 0.58 & 0.50 \\
\hline Hip-Troch BMD & 0.57 & 0.47 & 0.52 & 0.57 & 0.08 & 0.53 & 0.41 & 0.56 & 0.51 \\
\hline
\end{tabular}

\section{DESCRIPTION OF NEW PORTABLE BATTERY-POWERED DEVICE}

Based on results of the two studies presented above, a new device utilizing similar signal processing and parameters was designed and built. In contrast to the computer based and off-line nature of the device used previously, the new device, known as the $Q R T 2000$, is handheld, portable and is powered by 4 "AA" rechargeable batteries. It processes the data in largely real-time and displays the parameter estimates for immediate feedback to the user. The device is constructed around an Analog Devices DSP (ADSP2065LKS-240) operating at $60 \mathrm{MHz}$ (180 MFLOPS) and a 14-bit A/D converter that samples the ultrasound signal at $30 \mathrm{MHz}$. The overall structure is shown in Fig. 6, and the receiver circuit board about $2 \times 5$ inches -- undergoing final testing is shown in Fig. 7. The pulser is contained on a separate circuit board measuring about $2 \times 2$ inches (not shown), and produces a 300 volt 400 nanosecond signal that excites the source transducer. The processed data (ultrasound parameters) are output to a small LCD graphics display, and the overall control logic is provided by an embedded microcontroller. Fig. 8 shows a computer rendering of the $Q R T 2000$; the spacing of the two transducers in the rendering is 1.5 inches, and the overall height is about nine (9) inches. 


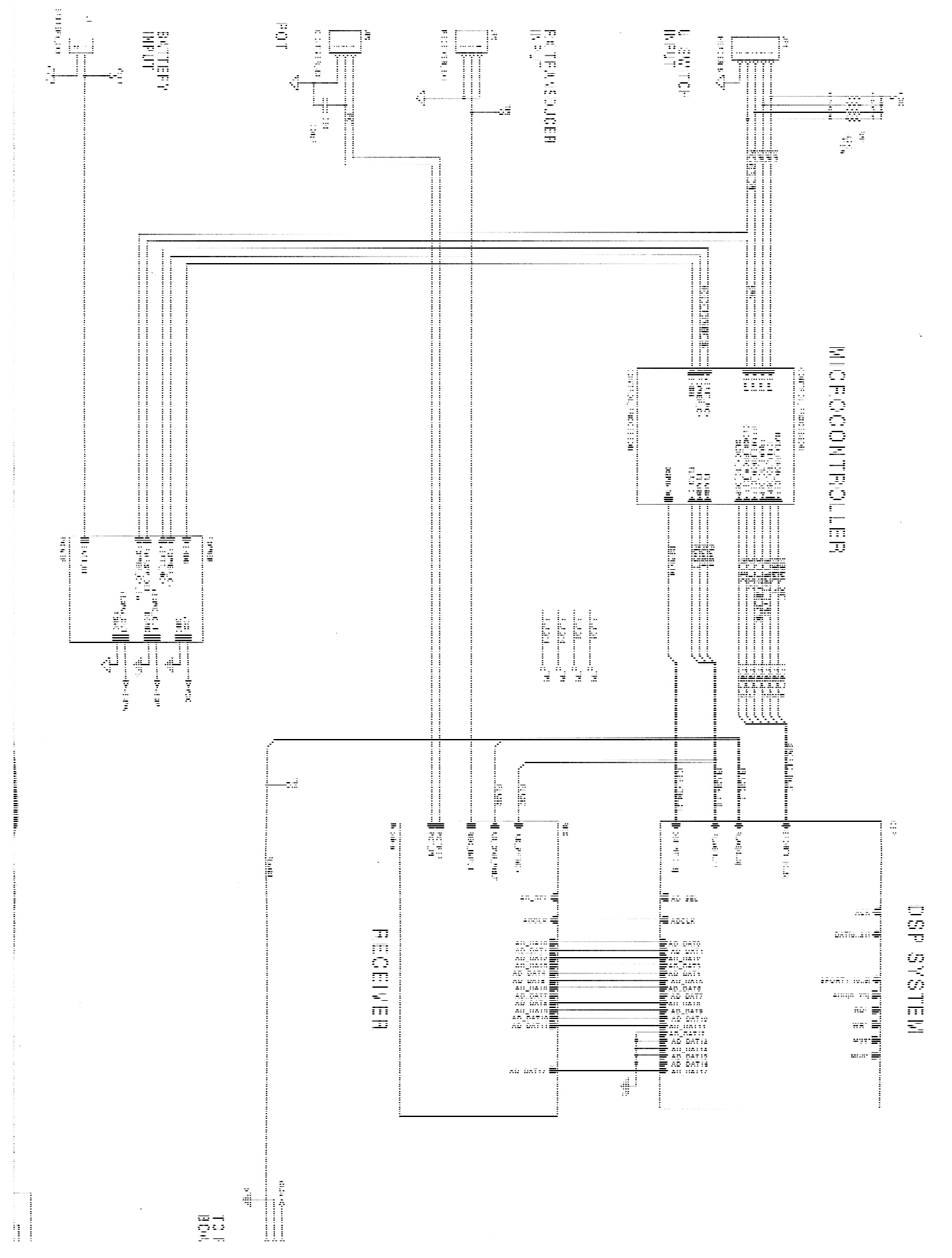

Figure 6. Schematic diagram of electronic components of the $Q R T 2000$. 


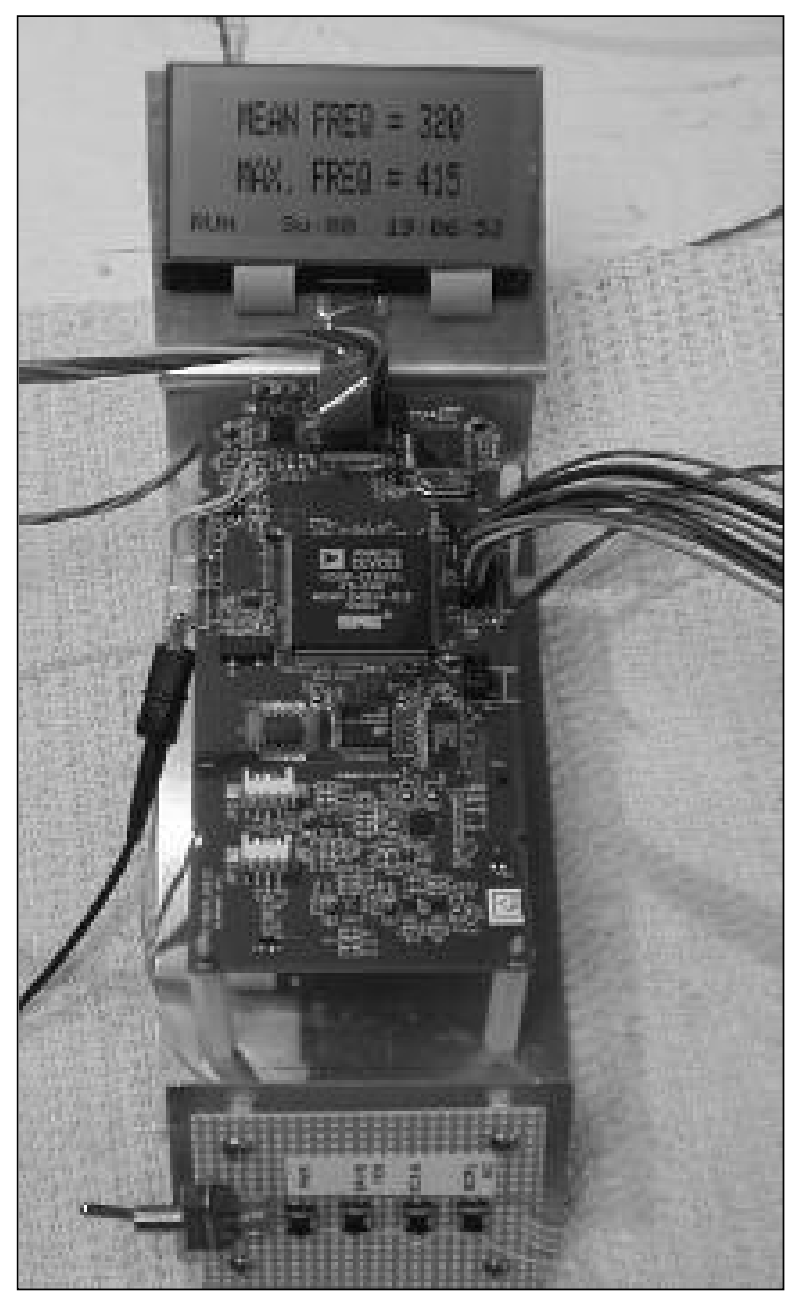

Figure 7. Receiver processing circuit board under test. This board contains the DSP, A/D converter and microcontroller.

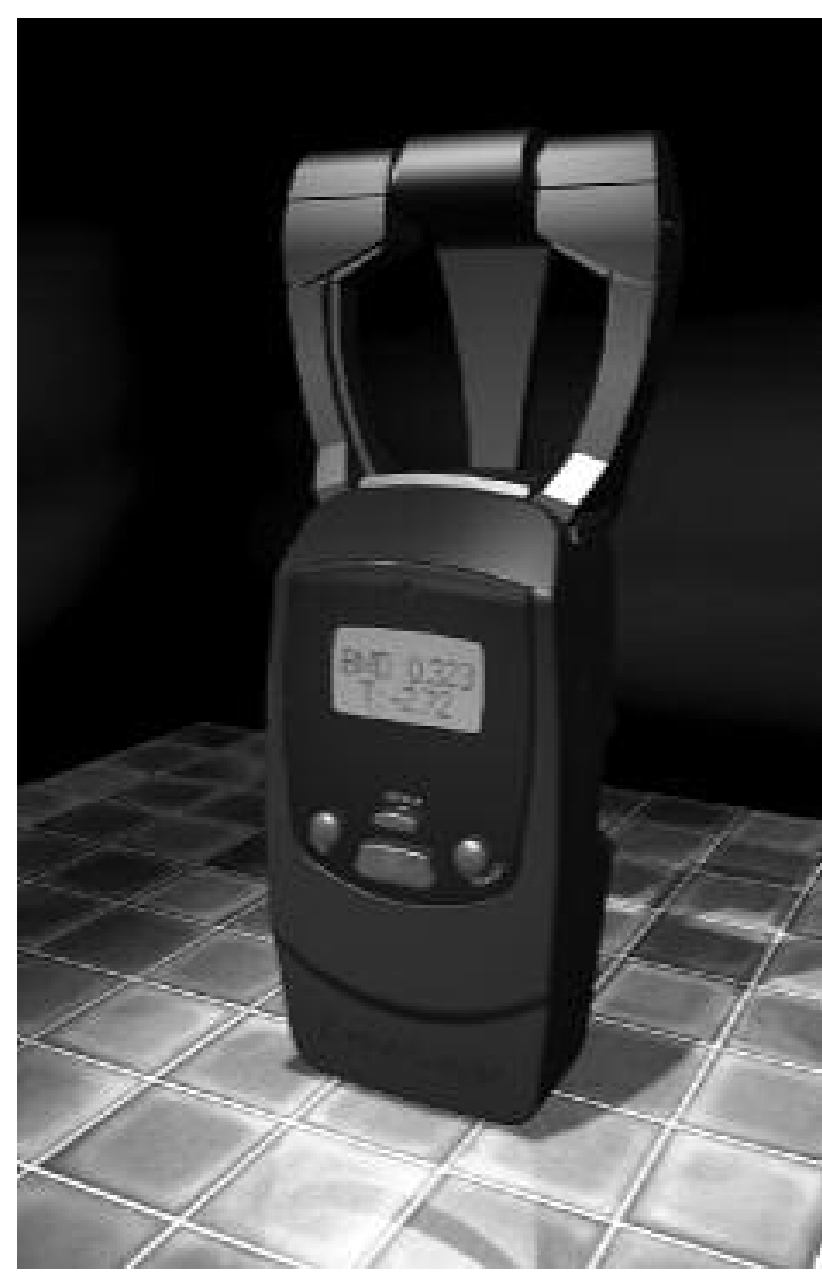

Figure 8. Computer rendering of the $Q R T 2000$ ultrasound bone assessment device. The overall height is about 9 inches, while the distance between the transducers as shown is 1.5 inches.

The CyberLogic QRT 2000 ultrasound device is currently being tested in clinical studies. These studies are including subjects who have had an osteoporotic fracture to determine the usefulness of the $Q R T 2000$ for estimating fracture risk, as well as BMD. It is expected that the device will offer superior performance over that currently available, and that, combined with the unit's portability and hand-held nature, will make it widely utilized for non-invasive bone assessment. In summary, the QRT 2000 offers: (1) a real-time system with on-line feedback which may open up an entire new protocol for the user that can improve reproducibility that may more accurately assess bone changes in osteoporosis and during treatment -- by being able to focus in on specific regions of interest in an adaptive fashion; (2) novel signal processing algorithms less sensitive to artifacts and ability to extract more information from the signals than standard algorithms; and (3) battery-powered portability and hand-held inexpensive design which should lead to improved healthcare worldwide, in terms of osteoporosis diagnosis and management. 


\section{REFERENCES}

1. JJ Kaufman, RS Siffert, "Non-invasive Assessment of Bone Integrity," in Bone Mechanics Handbook, SC Cowin, Ed, pp. 34.1-34.25, CRC Press, Boca Raton, 2001.

2. AM Parfitt, "Trabecular bone architecture in the pathogenesis and prevention of fracture," Am J Med, 82(1B), pp. 68-72, 1987.

3. RS Siffert, GM Luo, SC Cowin, JJ Kaufman, "Dynamical relationships of trabecular bone density, architecture and strength in a computational model of osteopenia," Bone, 18(2), pp. 197-206, 1996.

4. JJ Kaufman et al., "Review - Ultrasound Assessment of Bone," J Bone Min Research, 8(5), pp. 517-525, 1993.

5. GM Luo, JJ Kaufman, A Chiabrera, B Bianco, JH Kinney, D Haupt, JT Ryaby, RS Siffert, "Computational methods for ultrasonic bone assessment," Ultrasound in Medicine and Biology, 25(5), pp. 823-830, 1999.

6. JM Alves, JT Ryaby, RS Siffert, F Magee, JJ Kaufman, "Influence of marrow on ultrasonic velocity and attenuation, Calcif Tissue Int, 58, pp. 362-367, 1996.

7. JM Alves, W Xu, D Lin, RS Siffert, JT Ryaby, JJ Kaufman, "Ultrasonic assessment of human and bovine trabecular bone: a comparison study," IEEE Transactions on Biomedical Engineering, 43(3), pp. 249-258, 1996.

8. JJ Kaufman and AE Chiabrera "Ultrasonic Bone Assessment Apparatus and Method" United States Patent Number 5,651,363, Issued July 29, 1997.

9. AE Chiabrera and JJ Kaufman "Ultrasonic Bone Assessment Method and Apparatus" United States Patent Number 5,785,656, Issued July 28, 1998.

10. AE Chiabrera and JJ Kaufman "Ultrasonic Bone Assessment Method and Apparatus" United States Patent Number 5,879,301, Issued March 9, 1999.

\section{ACKNOWLEDGEMENTS}

This work was supported by Small Business Innovative Research Grant Numbers 1R43AR45150 and 2R44AR45150 from the National Institute of Arthritis and Musculoskeletal and Skin Diseases of the National Institutes of Health. The authors would also like to gratefully acknowledge the assistance of Dr. Jose Marcos Alves and Dr. James T. Ryaby in the acquisition of the in vitro data. 